\title{
Coordinated Depth Control of Multiple Autonomous Underwater Vehicles by Using Theory of Adaptive Sliding Mode
}

\author{
Yintao Wang $\mathbb{D}^{1,2}$ and Yani Zhang $\mathbb{D}^{1}$ \\ ${ }^{1}$ School of Marine Science and Technology, Northwestern Polytechnical University, Xian 710072, China \\ ${ }^{2}$ National Key Laboratory of Underwater Information Processing and Control, Xian, Shaanxi 710072, China \\ Correspondence should be addressed to Yintao Wang; wangyintao@nwpu.edu.cn
}

Received 16 July 2018; Revised 5 October 2018; Accepted 8 November 2018; Published 2 December 2018

Guest Editor: Zhaojie Ju

Copyright (C) 2018 Yintao Wang and Yani Zhang. This is an open access article distributed under the Creative Commons Attribution License, which permits unrestricted use, distribution, and reproduction in any medium, provided the original work is properly cited.

\begin{abstract}
This paper addresses the coordinated depth control problem of multiple autonomous underwater vehicles, which means to maneuver a group of underwater vehicles which move at the same depth synchronously. Firstly, a coordinated error of depth between vehicles and the common desired depth is defined by using extended graph theory in a distributed manner; then a deeppitch double loop control algorithm based on sliding mode is designed for each vehicle, by which each vehicle is driven to and move at the common depth coordinately. In particular, a pitch reference command is firstly calculated by the predefined coordinated depth error, which can be regarded as the outer loop control, and then, the input rudder angle for each vehicle is derived according to the pitch reference command being as the inner loop control. Considering the uncertainties of the model hydrodynamic parameters, an online parameter adaptive algorithm is introduced to improve the performance of the sliding mode control algorithm proposed. Simulations were performed to verify the theoretical results proposed.
\end{abstract}

\section{Introduction}

With the development of artificial intelligence, robots play more and more roles in our daily lives. The high demand of industrial applications and automation devices always needs the robot operate in a complex environment which is posing requirements for the system stability, safety, and strong challenges for the controller design [1-3]. As a typical underwater robot, Autonomous Underwater Vehicle (AUV) plays great roles in ocean exploring missions and even more in the field of militaries. In order to fulfill these applications, it is usually necessary to maneuver an AUV cruise at a fixed and expected depth; this task is always called the vertical plane control of AUV, which is a typical motion form in the field of marine crafts [4]. Many works have been done to develop strategies capable of depth control of a single AUV, mainly include fuzzy control [5], neural networks [6], and sliding mode $[7,8]$. Although significant progress has been made in the area, however, much work remains to be done to develop strategies for multiple AUVs. Considering the fact that current missions are getting more complex and AUV technology matures, it is common to require multiple AUVs to work cooperatively to solve these types of tasks with low cost, high adaptively, and easy maintenance.

Consensus theory and algorithms have proven to be effective tools to perform the network-wide distributed computation tasks such as computing aggregate quantities and functions over networks. There have been much works on consensus problems of first-order agents, such as consensus under time-varying topology [9], finite-time consensus [10], consensus over random networks [11], and asynchronous consensus [12]. Taking into account the fact that many vehicles such as AUVs considered in this paper and mobile robots are always controlled directly by their accelerations rather than by their velocities, hence it is also necessary to investigate consensus problems of second-order agents. In [13, 14], the authors studied conditions on the interaction graph and the control gains for two different consensus algorithms to ensure agreement on both positions and velocities. Despite significant progress has been made in aforementioned works, much work remains to be done for the AUV systems due to the fact that the dynamics of AUV are often complex and cannot be simply ignored or drastically simplified for control design purpose. In the field of marine vehicles, authors in 
[15] proposed a synchronized path following controller for fully actuated surface ships by using passivity theory. A decentralized formation controller was derived in [16], which deals with the cooperative problem of fully actuated surface vehicles with considering the influencing of the sea currents and model uncertainty. Even all the results proposed in the above woks are decentralized because each member of the group only needs to exchange the necessary information under local interactions, but all of them assumed that there is a global reference speed for the whole group which should be known to all the team members. In this sense, the controller proposed is not distributed because there is a global vector which should be known to all of them.

Motivated by the ideas of consensus tracking and aforementioned works, we consider the truly distributed depth coordinated control problem of AUVs in this proposal. By using extended graph theories, the coordinated depth error between each AUV and the common desired depth was defined firstly, in which we only need the common desired depth be available to one subset of AUVs by local interactions, so the strategy is truly distributed and this can be regarded as our first contribution. Moreover, a double loop based on adaptive sliding mode control algorithm for each AUV was derived with considering the model uncertainties of each AUV, and each AUV can move at the same depth coordinately by using the algorithm proposed. Furthermore, the control parameters used in the algorithm proposed are obtained from the kinematic model of the AUV and the expected dynamic characteristics of each link; except for the initial value of the model parameter vector, all other parameters are irrelevant to the AUV, so the strategy proposed has good application values.

The remainder of this paper is organized as follows: in Section 2, models of the vehicles considered in this work and extended graph theory which will be used throughout this paper are described. We also presented the basic principles of the synchronized depth control by defining a coordinated error vector novelty in this section. Sections 3 and 4 especially present the procedures of designing the sliding mode control for the depth tracking with and without considering the model uncertainties. In Section 5, simulation examples are executed to validate the effectiveness of the strategy proposed. Finally, conclusions and future works are summarized and discussed in Section 6.

\section{Problem Formulation}

2.1. AUV Model in Vertical Plane. The six degrees of freedom dynamic model of AUV can be decomposed into vertical plane motion and horizontal plane motion independently if some coupling constraints are satisfied. The depth control considered in this paper is a typical freedom in vertical plane, so before going on, we will give the AUV model in vertical plane borrowed from [4]

$$
\begin{aligned}
\dot{\boldsymbol{\eta}}_{v} & =\mathbf{R}_{v}(\theta) \mathbf{v}_{v} \\
\mathbf{M}_{v} \dot{\mathbf{v}}_{v}+\mathbf{C}_{v}\left(\mathbf{v}_{v}\right) \mathbf{v}_{v}+\mathbf{D}_{v}\left(\mathbf{v}_{v}\right) \mathbf{v}_{v}+\mathbf{g}_{v}(\theta) & =\boldsymbol{\tau}_{v},
\end{aligned}
$$

where $\boldsymbol{\eta}_{v}=\left[\begin{array}{lll}x & z & \theta\end{array}\right]^{T}$ denotes the position and pitch of AUV in earth-fixed frame $\{I\}$ and $\mathbf{v}_{v}=\left[\begin{array}{lll}u & w & q\end{array}\right]^{T}$ represents the speed in body-fixed frame $\{b\}$ with $\mathbf{R}_{v}(\theta)$ ) being the transformation matrix from frame $\{b\}$ to $\{I\} . \mathbf{M}_{v}$, $\mathbf{C}_{v}\left(\mathbf{v}_{v}\right), \mathbf{D}_{v}\left(\mathbf{v}_{v}\right)$, and $\mathbf{g}_{v}\left(\mathbf{v}_{v}\right)$ are the inertial matrix, Coriolis force matrix, damping matrix and gravity, and buoyancy generalized force vector with the following form, respectively:

$$
\begin{aligned}
& \mathbf{R}_{v}(\theta)=\left[\begin{array}{ccc}
\cos \theta & \sin \theta & 0 \\
-\sin \theta & \cos \theta & 0 \\
0 & 0 & 1
\end{array}\right], \\
& \mathbf{M}_{v}=\left[\begin{array}{ccc}
m-X_{\dot{u}} & 0 & 0 \\
0 & m-Z_{\dot{w}} & 0 \\
0 & 0 & I_{y y}-M_{\dot{q}}
\end{array}\right] \text {, } \\
& \mathbf{C}_{v}\left(\mathbf{v}_{v}\right) \\
& =\left[\begin{array}{ccc}
0 & 0 & \left(m-Z_{\dot{w}}\right) w \\
0 & 0 & -\left(m-X_{\dot{u}}\right) u \\
-\left(m-Z_{\dot{w}}\right) w & \left(m-X_{\dot{u}}\right) u & 0
\end{array}\right], \\
& \mathbf{D}_{v}\left(\mathbf{v}_{v}\right) \\
& =\left[\begin{array}{ccc}
-X_{u} & 0 & 0 \\
0 & -Z_{w} & 0 \\
0 & 0 & -M_{q}
\end{array}\right] \\
& +\left[\begin{array}{ccc}
-X_{|u| u}|u| u & 0 & 0 \\
0 & -Z_{|w| w}|w| & 0 \\
0 & 0 & -M_{|q| q}|q|
\end{array}\right] \\
& \mathbf{g}_{v}(\theta)=\left[\begin{array}{c}
(W-B) \sin \theta \\
-(W-B) \cos \theta \\
\left(z_{g} W-z_{b} B\right) \sin \theta+\left(x_{g} W-x_{b} B\right) \cos \theta
\end{array}\right] \text {, } \\
& \boldsymbol{\tau}_{v}=\left[\begin{array}{lll}
X & Z & M
\end{array}\right]^{T}=\left[\begin{array}{lll}
T & Z_{\delta} \delta_{e} & M_{\delta} \delta_{e}
\end{array}\right]^{T} \text {, }
\end{aligned}
$$

where $I_{y y}-M_{\dot{q}}>0$ is the combined moment of inertia with additional mass produced and the rotation inertia of the $y$-axis and $M_{\delta}$ is the rotation torque coefficient produced by rudder angle $\delta_{e}$. Due to the fact that when AUV makes down steering which will produce the negative pitch angular acceleration $\dot{q}$, therefore, $M_{\delta}<0 . \tau_{v}$ denotes the control force and torque for AUV which will be designed in following sections to enable each AUV move at the desired depth.

From the above formulations, we can find that the diving equations of AUV motion should include the heave velocity $w$, the angular velocity in pitch $q$, the pitch angle $\theta$, and the depth $z$. Assume that the forward speed is constant and that the sway and yaw modes can be neglected, this suggests 
the following simplified equations of AUV motion in vertical plane as

$$
\begin{aligned}
\dot{z}= & -u \sin \theta+w \cos \theta \\
\dot{\theta}= & q \\
\left(I_{y y}-M_{\dot{q}}\right) \dot{q}= & \left(X_{\dot{u}}-Z_{\dot{w}}\right) u w+M_{q} q+M_{|q| q} q|q| \\
& +G z_{G} \sin \theta+G x_{G} \cos \theta+M_{\delta} \delta_{e} \\
& +n_{\theta}^{\prime},
\end{aligned}
$$

where $n_{\theta}^{\prime}$ denotes the bounded total modeling errors and external disturbance. Based on parameterized linearization techniques, the dynamic equation of pitch angular velocity $q$ can be rewritten as

$$
\begin{aligned}
\dot{q}= & a_{1} u w+a_{2} q+a_{3} q|q|+a_{4} \sin \theta+a_{5} \cos \theta+b \delta_{e} \\
& +n_{\theta},
\end{aligned}
$$

where

$$
\begin{aligned}
& a_{1}=\frac{\left(X_{\dot{u}}-Z_{\dot{w}}\right)}{\left(I_{y y}-M_{\dot{q}}\right)}, \\
& a_{2}=\frac{M_{q}}{\left(I_{y y}-M_{\dot{q}}\right)}, \\
& a_{3}=\frac{M_{|q| q}}{\left(I_{y y}-M_{\dot{q}}\right)}, \\
& a_{4}=\frac{G z_{G}}{\left(I_{y y}-M_{\dot{q}}\right)}, \\
& a_{5}=\frac{G x_{G}}{\left(I_{y y}-M_{\dot{q}}\right)}, \\
& b=\frac{M_{\delta}}{\left(I_{y y}-M_{\dot{q}}\right)}, \\
& n_{\theta}=\frac{n_{\theta}^{\prime}}{\left(I_{y y}-M_{\dot{q}}\right)} .
\end{aligned}
$$

To be noted that, all the AUVs considered will be isomorphic in this paper, so for the sake of convenience, we omitted the index of the AUV number in the presentation of the model, and furthermore, in the implementing of the controller designing, we will make the following assumptions.

Assumption 1. The depth $z$ can be measured by a pressure meter and the pitch angle $\theta$ can be measured by an inclinometer while the pitch rate $q$ requires a rate gyro or a rate sensor. We also assumed that the velocities in the vertical plane can be measured by using a Doppler log for directly obtaining velocity measurements.

Assumption 2. The external disturbance $n_{\theta}^{\prime}$ is bounded by an unknown upper bound, and $n_{\theta}$ is bounded apparently. We

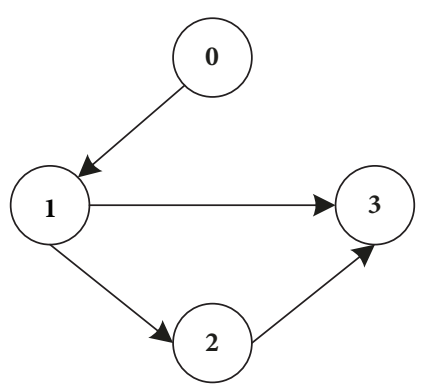

Figure 1: Example graph and the according Laplacian matrix.

assumed that $n_{\theta i} \leq n_{i}$, where $N=\left[n_{0}, n_{1}, \cdots, n_{n}\right]^{T}$ to be the unknown upper bound of disturbance [17].

2.2. Extended Communication Graph. Assumed that there are $n$ AUVs in the group, it is a conventional way to model the interactions between them using directed graph adjacency matrix and topology graphs [18]. Firstly, we introduce a virtual AUV, denoted as $v_{0}$, which specifically represents the desired state. For the $n$ AUVs system, the graph $G=\left(\mathrm{V}, A^{*}\right)$ contains a set of nodes $\mathrm{V}=\left\{v_{0}, v_{1}, \cdots, v_{n}\right\}$, and an adjacent matrix $A^{*}=\left[a_{i j}^{*}\right]$, where $a_{i j}^{*}=1$ indicates that the $i$ th AUV can obtain state information from $j$ th, otherwise $a_{i j}^{*}=0$. Then we define a degree matrix $\Delta$ with the elements $\delta_{i i}=\Sigma_{j} a_{i j}^{*}$. The Laplacian matrix of graph $G$ is expressed as $\bar{L}=\Delta-A^{*}$ and the normalized Laplacian matrix of $G$ is $L=I-A$, where $A$ is the normalization of $a_{i j}=a_{i j}^{*} / \delta_{i i}$ when $\delta_{i i} \neq 0$; otherwise $a_{i j}=a_{i j}^{*}$ [19]. If we add one virtual AUV to the group, then we call the graph $G$ as extended communication graph. An example of extended communication graph is given below; where Figure 1 is a topology graph with four nodes which contains three AUVs and one virtual AUV, its adjacency matrix, degree matrix, normalized adjacency matrix, Laplacian matrix, and normalized Laplacian matrix are, respectively,

$$
\begin{aligned}
A^{*} & =\left[\begin{array}{llll}
0 & 0 & 0 & 0 \\
1 & 0 & 0 & 0 \\
0 & 1 & 0 & 0 \\
0 & 1 & 1 & 0
\end{array}\right], \\
\Delta & =\left[\begin{array}{llll}
0 & 0 & 0 & 0 \\
0 & 1 & 0 & 0 \\
0 & 0 & 1 & 0 \\
0 & 0 & 0 & 2
\end{array}\right], \\
A & =\left[\begin{array}{llll}
0 & 0 & 0 & 0 \\
1 & 0 & 0 & 0 \\
0 & 1 & 0 & 0 \\
0 & \frac{1}{2} & \frac{1}{2} & 0
\end{array}\right],
\end{aligned}
$$




$$
\begin{aligned}
\bar{L} & =\left[\begin{array}{cccc}
0 & 0 & 0 & 0 \\
-1 & 1 & 0 & 0 \\
0 & -1 & 1 & 0 \\
0 & -1 & -1 & 2
\end{array}\right], \\
L & =\left[\begin{array}{cccc}
1 & 0 & 0 & 0 \\
-1 & 1 & 0 & 0 \\
0 & -1 & 1 & 0 \\
0 & -\frac{1}{2} & -\frac{1}{2} & 1
\end{array}\right] .
\end{aligned}
$$

Because the virtual AUV can not get any information from others, the row sum of the matrix $A$ is equal to 1 , except for the first row. It is obvious that all the diagonal elements of $L$ are 1 ; we can know that $L$ is a diagonal dominant matrix, positive define, and reversible; its inverse matrix will satisfy with $L^{-1}=\sum_{i=0}^{n} A^{i}[20]$.

2.3. Basic Idea of Sliding Mode Control. Consider the following second-order controlled object [21]:

$$
\ddot{x}(t)=f(x, t)+b u(t)+d(t),
$$

where $f(x, t)$ is nonlinear term, $d(t)$ is external disturbances, and $u(t)$ is the control input for the system.

Define tracking error as

$$
e(t)=x(t)-x_{d}
$$

where $x_{d}$ is desired state value.

Sliding surface vector is defined as follows:

$$
s(t)=\lambda e(t)+\dot{e}(t),
$$

where $\lambda>0$ and satisfies Hurwitz condition. Then the basic idea of sliding mode control is how to derive the input $u(t)$ determined by $s(t)$ for the system to make $s(t)$ and $e(t)$ go to zero asymptotically.

\subsection{Basic Principles of Coordinated Depth Control. Assume} that there are $n$ AUVs in the group, and in the following sections, we will use a subscript $i$ to denote the index number of AUV. For multiple AUVs, we redefine each parameter as follows: $z=\left[z_{0}, z_{1}, \cdots, z_{n}\right]^{T}$ is the depth of $n$ AUVs, respectively, and $z_{0}$ means depth of the virtual AUV, $u=$ $\left[u_{0}, u_{1}, \cdots, u_{n}\right]^{T}$ is the velocity of $n$ AUVs along $\mathrm{x}$-axis, $w=\left[w_{0}, w_{1}, \cdots, w_{n}\right]^{T}$ is the velocity of $n$ AUVs along $\mathrm{z}$ axis, $\theta=\left[\theta_{0}, \theta_{1}, \cdots, \theta_{n}\right]^{T}$ is the pitch angle of $n$ AUVs, $q=\left[q_{0}, q_{1}, \cdots, q_{n}\right]^{T}$ is the angular velocity around y-axis, and $\delta_{e}=\left[\delta_{e 0}, \delta_{e 1}, \cdots, \delta_{e n}\right]^{T}$ is the rudder angle. To make each AUV track a common desired depth synchronously, the tracking error for $i$ th vehicle will be defined as

$$
\widetilde{z}_{i}=\sum_{j=0}^{n} a_{i j}\left(z_{i}-z_{j}\right),
$$

where $a_{i j}$ is the $i j$ th element of the normalized adjacent matrix $A$ induced by the AUVs. According to the definition of normalized adjacent matrix in Section 2, the tracking error can also be described as

$$
\widetilde{z}_{i}=z_{i}-z_{d i}
$$

where $z_{d i}=\sum_{j=0}^{n} a_{i j} z_{j}$. Denote desired depth as $z_{d}=$ $\left[z_{d 0}, z_{d 1}, \cdots, z_{d n}\right]^{T}$; then the collective depth tracking error of $n$ AUVs can be described as

$$
\widetilde{z}=z-z_{d}
$$

where $\widetilde{z}=\left[\widetilde{z}_{0}, \widetilde{z}_{1}, \cdots, \widetilde{z}_{n}\right]^{T}$. It is known from (8) that the dynamics of the depth can be also described as

$$
\dot{z}=-v \sin (\theta-\alpha)
$$

where $v=\operatorname{diag}\left(v_{0}, v_{1}, \cdots, v_{n}\right)$ with $v_{i}=\sqrt{u_{i}^{2}+w_{i}^{2}},(i=$ $0,1, \cdots, n), \alpha=\left[\alpha_{0}, \alpha_{1}, \cdots, \alpha_{n}\right]^{T}$ with $\alpha_{i}=\arctan \left(-w_{i} /\right.$ $\left.u_{i}\right),(i=0,1, \cdots, n)$, and $\sin (\theta-\alpha)=\left[\sin \left(\theta_{0}-\alpha_{0}\right), \sin \left(\theta_{1}-\right.\right.$ $\left.\left.\alpha_{1}\right), \cdots, \sin \left(\theta_{n}-\alpha_{n}\right)\right]^{T}$.

Assume that the depth tracking error will evolve with the following dynamics:

$$
\dot{\tilde{z}}+\lambda_{z} \tilde{z}=0
$$

where $\lambda_{z}=\operatorname{diag}\left(\lambda_{z 0}, \lambda_{z 1}, \cdots, \lambda_{z n}\right)$ with $\lambda_{z i}$ being the positive gains. Then the tracking error $\widetilde{z}$ will converge to zero exponentially at the rate of $\lambda_{z}$. This assumption is commonly used for error dynamic equations. Consider the following first-order homogeneous linear differential equation and its general solutions:

$$
\begin{aligned}
\frac{d y}{d x}+P(x) y & =0, \\
y & =C e^{-\int P(x) d x},
\end{aligned}
$$

where $C$ is a constant. Then solution (21) will be determined by the initial condition of (20) and $P$.

That means

$$
\widetilde{z}=C e^{-\int \lambda_{z} d t}=C e^{-\lambda_{z} t},
$$

so the depth error $\widetilde{z}$ converges to zero when $t$ tends to infinity.

Denote the desired pitch angle for each AUV as $\theta_{f}=$ $\left[\theta_{f 0}, \theta_{f 1}, \cdots, \theta_{f n}\right]^{T}$, then we can have

$$
-v \sin \left(\theta_{f}-\alpha\right)=\dot{z}_{d}-\lambda_{z}\left(z-z_{d}\right)
$$

By simply calculations we can get

$$
\theta_{f}=-\arcsin \left(\nu^{-1}\left(\dot{z}_{d}-\lambda_{z}\left(z-z_{d}\right)\right)\right)+\alpha,
$$

assuming that $\nu^{-1}\left(\dot{z}_{d}-\lambda_{z}\left(z-z_{d}\right)\right)=\mu$, then $\arcsin (\mu)=$ $\left[\arcsin \left(\mu_{0}\right), \arcsin \left(\mu_{1}\right), \cdots, \arcsin \left(\mu_{n}\right)\right]^{T}$.

Here we require $\mu_{i} \leq 1$; otherwise, the objective of depth control cannot be achieved. In physical sense, this 


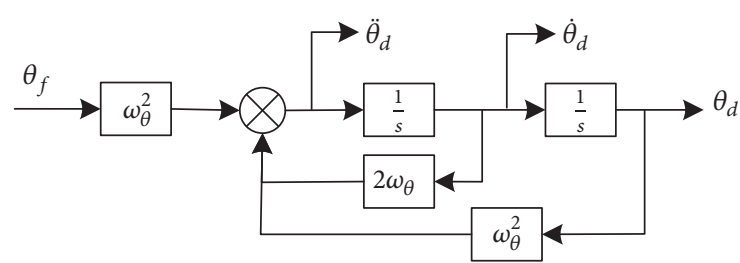

FIGURE 2: Second-order system structure block diagram.

inequality constraint means the expectation of the depth changing rate of each AUV can not be greater than its total speed; moreover, consider the fact that we have assumed a linearization hypothesis of a pitching angle being as a small angle before, then characteristic of $\arcsin x=x$ could be used here. Furthermore, by putting some saturation limit on $\left(z-z_{d}\right)$ we will have

$$
\theta_{f}=-v^{-1}\left(\dot{z}_{d}-\lambda_{z} \Phi_{z} s a t\left(\frac{\left(z-z_{d}\right)}{\Phi_{z}}\right)\right)+\alpha,
$$

and the coefficient $\Phi_{z}=\left[\Phi_{z 0}, \Phi_{z 1}, \cdots, \Phi_{z n}\right]^{T}$ determines the range of the depth deviation limit, that is, when the depth deviation of $i$ th AUV is greater than $\Phi_{z i}$, the pitch angle instruction will no longer be increased.

To make the desired pitch angle for each AUV to be more smooth, we will introduce a second-order filter to represent the dynamic relationship between $\theta_{d}$ and $\theta_{f}$ as

$$
\omega_{\theta}^{2} \theta_{d}+2 \omega_{\theta} \dot{\theta}_{d}+\ddot{\theta}_{d}=\omega_{\theta}^{2} \theta_{f},
$$

where $\omega_{\theta}=\operatorname{diag}\left(\omega_{\theta 0}, \omega_{\theta 1}, \cdots, \omega_{\theta n}\right)$ with $\omega_{\theta i}>0$ and $\theta_{d}=$ $\left[\theta_{d 0}, \theta_{d 1}, \cdots, \theta_{d n}\right]^{T}$ will be the desired pitch angle output for each AUV. The block diagram of this filter was shown in Figure 2.

As so far, the structure of depth control considered in this paper can be shown in the Figure 3, where we put forward a deep-pitch double loop control strategy with pitch angle controller as the inner loop and depth controller as the outer loop [22]. The desired common depth $z_{d}$ is initialized by the outer loop, and the according desired pitch angle $\theta_{d}$ is determined by the desired depth which means the input $\theta_{d}$ of the inner loop to be the output of the outer loop control, and the entire control system constitutes a closed-loop feedback structure.

\section{Coordinated Depth Control without Model Uncertainties Based on Sliding Mode}

To make the attitude of each AUV track the desired attitude $\theta_{d 0}$ derived in Section 2 before synchronously, define the $i$ th attitude tracking error as

$$
e_{i}=\sum_{j=0}^{n} a_{i j}\left(\theta_{i}-\theta_{j}\right) .
$$

According to the definition of normalized adjacent matrix $A$, this attitude tracking error also can be described as

$$
e_{i}=\theta_{i}-\theta_{d i},
$$

where $\theta_{d i}=\sum_{j=0}^{n} a_{i j} \theta_{j}$; as an example of Figure 1 , the synchronized attitude error will be

$$
\begin{aligned}
& e_{0}=0 \\
& e_{1}=\theta_{1}-\theta_{d 0} \\
& e_{2}=\theta_{2}-\theta_{1} \\
& e_{3}=\theta_{3}-\frac{1}{2} \theta_{2}-\frac{1}{2} \theta_{1},
\end{aligned}
$$

so the attitude deviation of $n$ AUVs can be defined as

$$
e=\theta-\theta_{d},
$$

where $e=\left[e_{0}, e_{1}, \cdots, e_{n}\right]$; according to the definition of normalized Laplacian L, we can obtain

$$
e=L \theta \text {. }
$$

Nextly, we will show how to derive the control input for each AUV by using the coordinated error defined by (30). Before going on, define the linearized parameter vector $\mathbf{a}=\left[\begin{array}{lllll}\mathrm{a}_{1} & \mathrm{a}_{2} & \mathrm{a}_{3} & \mathrm{a}_{4} & \mathrm{a}_{5}\end{array}\right]$ and $\mathbf{Y}=\left[\begin{array}{lllll}y_{1} & y_{2} & y_{3} & y_{4} & y_{5}\end{array}\right]=$

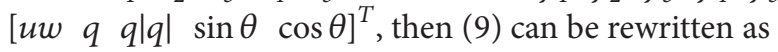

$$
\dot{q}=\mathbf{a} Y+b \delta_{e}+n_{\theta} .
$$

According to the introduction of Section 2, define the sliding mode vector as

$$
S=\dot{e}+\Lambda e,
$$

where $S=\left[s_{0}, s_{1}, \cdots, s_{n}\right]^{T}$ and $\Lambda=\operatorname{diag}\left(\lambda_{0}, \lambda_{1}, \cdots, \lambda_{n}\right)$ is a design parameter with $\lambda_{i}>0$.

By simply calculations we will get the derivatives of (33):

$$
\dot{S}=\dot{q}-\ddot{\theta}_{d}+\Lambda L q .
$$

Substituting (32) into (34), we can get

$$
\dot{S}=\mathbf{a Y}+b \delta_{e}+n_{\theta}-\ddot{\theta}_{d}+\Lambda L q .
$$

Choose the exponential reaching law as

$$
\dot{S}=-T S-K \operatorname{sgn}(S),
$$

where $T=\operatorname{diag}\left(t_{0}, t_{1}, \cdots, t_{n}\right)$ and $K=\operatorname{diag}\left(k_{0}, k_{1}, \cdots, k_{n}\right)$. Then the control law for AUVs can be proposed as

$$
\begin{aligned}
& \delta_{e} \\
& =c\left[-T S-K \operatorname{sgn}(S)-\mathbf{a Y}-N \operatorname{sgn}(S)+\ddot{\theta}_{d}-\Lambda L q\right],
\end{aligned}
$$

where $c=\operatorname{diag}\left(c_{0}, c_{1}, \cdots, c_{n}\right)=b^{-1}$ with $c_{i}>0$.

Theorem 3. Consider the system composed by a fleet of $n$ AUVs of the form (8), guided by the slide mode control laws (37) with the sliding mode surface defined by (33). Then, the control system proposed solves the coordinated depth problem; that is, all the AUVs will move at the same common desired depth when this referred depth information can be available to at least one AUV and the graph induced by the AUVs has a directed spanning tree. 


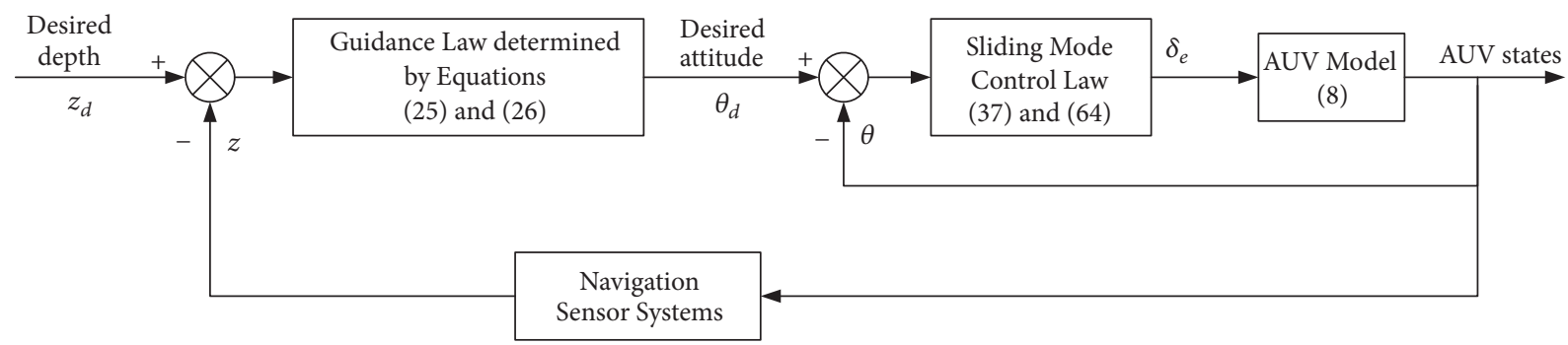

FIGURE 3: The structure of depth control system.

Proof. Consider the Lyapunov function candidate:

$$
V=\frac{1}{2} S^{T} L^{-1} S .
$$

The derivative of $V$ is

$$
\dot{V}=S^{T} L^{-1} \dot{S}
$$

Substituting (34) into (39), we will have

$$
\dot{V}=S^{T} L^{-1}\left[\mathbf{a Y}+b \delta_{e}+n_{\theta}-\ddot{\theta}_{d}+\Lambda L q\right],
$$

and continuously substituting control law (37) into (40), we will get

$$
\begin{aligned}
\dot{V} & =S^{T} L^{-1}[\mathbf{a Y} \\
& +\left[-T S-K \operatorname{sgn}(S)-\mathbf{a Y}-N \operatorname{sgn}(S)+\ddot{\theta}_{d}-\Lambda L q\right] \\
& \left.+n_{\theta}-\ddot{\theta}_{d}+\Lambda L q\right] .
\end{aligned}
$$

Simply manipulations will yield

$$
\dot{V}=S^{T} L^{-1}\left[-T S-K \operatorname{sgn}(S)-N \operatorname{sgn}(S)+n_{\theta}\right] .
$$

According to Assumption 2, we know that $-N \operatorname{sgn}(S)+$ $n_{\theta}<0$, thus we can get

$$
\dot{V} \leq-S^{T} L^{-1} T S-S^{T} L^{-1} K \operatorname{sgn}(S) .
$$

According to Lyapunov stability theorem, the following two stability conditions will be hold as

$$
\begin{aligned}
& \lim _{\left|s_{i}\right| \longrightarrow \infty} V=\infty, \\
& \dot{V}_{i}<0, \quad\left(s_{i} \neq 0\right) .
\end{aligned}
$$

So $S$ and $e$ will converge to zero asymptotically. Consequently, we will show that when the desired common depth information can only be obtained by at least one of the AUVs, all the AUVs can track this desired state coordinately; in order to do this, define the error between $i$ th AUV and the desired attitude angle as

$$
\widetilde{\theta}_{i}=\theta_{i}-\theta_{d 0}
$$

and the $i$ th sliding mode surface as

$$
s_{i}=\dot{e}_{i}+\lambda_{i} e_{i}=\left(\dot{\theta}_{i}-\sum_{j=0}^{n} a_{i j} \dot{\theta}_{j}\right)+\lambda_{i}\left(\theta_{i}-\sum_{j=0}^{n} a_{i j} \theta_{j}\right),
$$

consequently introducing the auxiliary variables as

$$
\xi_{i}=\dot{\theta}_{i}+\lambda_{i} \theta_{i}
$$

Substituting (48) into (47), the sliding mode surface can be rewritten as

$$
s_{i}=\xi_{i}-\sum_{j=0}^{n} a_{i j} \xi_{j}
$$

then we can have

$$
\widetilde{\xi}_{i}=\xi_{i}-\xi_{0}=\sum_{j=0}^{n} a_{i j} \xi_{j}+s_{i}-\xi_{0},
$$

and rewrite (50) in a vector form with

$$
\tilde{\xi}=A \xi+S-\xi_{0} \mathbf{1}
$$

where $\mathbf{1}=[1,1, \cdots, 1]^{T}$ and $A$ is the corresponding normalized weighted adjacency matrix. Considering the fact that the sum of first row elements of $A$ is 0 and other rows is 1 which means $A[1,1, \cdots, 1]^{T}=[0,1,1, \cdots, 1]^{T}$, then we will have

$$
\begin{aligned}
\widetilde{\xi} & =A \xi+S-\xi_{0} \mathbf{1} \\
& =A\left(\widetilde{\xi}+\xi_{0} \mathbf{1}\right)+S+[1,0,0, \cdots, 0]^{T} \xi_{0}-\xi_{0} \mathbf{1} \\
& =A \widetilde{\xi}+[0,1, \cdots, 1]^{T} \xi_{0}+S+[1,0, \cdots, 0]^{T} \xi_{0}-\xi_{0} \mathbf{1} \\
& =A \widetilde{\xi}+S
\end{aligned}
$$

which means

$$
\widetilde{\xi}=(I-A)^{-1} S
$$

according to Section 2, we know that the Laplace matrix of the system is positive definite and reversible. So (53) will become

$$
\widetilde{\xi}=L^{-1} S
$$


According to (48), the time derivative of $\widetilde{\theta}_{i}$ is

$$
\dot{\tilde{\theta}}_{i}=\dot{\theta}_{i}-\dot{\theta}_{d 0}=a_{\theta} \widetilde{\theta}_{i}+\widetilde{\xi}_{i}
$$

where $a_{\theta}=-\lambda_{i}$, and the vector form of (55) is

$$
\dot{\tilde{\theta}}=\bar{a}_{\theta} \widetilde{\theta}+\tilde{\xi}
$$

where $\bar{a}_{\theta}=-\Lambda$. By simply manipulations, we can get

$$
\dot{\tilde{\theta}}=\bar{a}_{\theta} \widetilde{\theta}+L^{-1} S,
$$

Lemma 4. For the time constant $T_{1}$, define $s_{\max , i}(t)=$ $\max _{i} \sup _{0 \leq \tau \leq t}\left|s_{i}\right|$; the following inequalities will be hold:

$$
\|\widetilde{\theta}(t)\| \leq \bar{\kappa}\|\tilde{\theta}(0)\|+\frac{k_{0}}{\lambda_{0}}\left(\beta_{s}\left(T_{1}\right)+\beta_{s T_{1}}\right),
$$

where $\lambda_{\max }$ is the maximum eigenvalue of $L^{-1}, \beta_{s}(t)=$ $N \lambda_{\max } s_{\max , i}(t), \beta_{s T_{1}}=N \lambda_{\text {max }} \sup _{T_{1}<t} s_{\text {max }, i}(t)$, and $\bar{\kappa}=$ $k_{0} e^{-\lambda_{0} t} \geq\left\|e^{\bar{a}_{\theta} t}\right\|, k_{0}>0, \lambda_{0}>0$.

Proof. The solution of (57) can be derived as

$$
\tilde{\theta}(t)=\tilde{\theta}(0) e^{\bar{a}_{\theta} t}+\int_{0}^{t} e^{\bar{a}_{\theta}(t-\tau)} L^{-1} S d \tau
$$

Considering the fact that the Laplace matrix of system $L$ is positive definite and $L^{-1}$ is positive definite too. According to triangle inequality axiom of the matrix norm

$$
\|A+B\| \leq\|A\|+\|B\|,
$$

we will have

$$
\begin{aligned}
\|\widetilde{\theta}(t)\| \leq & \left\|\widetilde{\theta}(0) e^{\bar{a}_{\theta} t}\right\|+\left\|\int_{0}^{t} e^{\bar{a}_{\theta}(t-\tau)} L^{-1} S d \tau\right\| \\
\leq & k_{0} e^{-\lambda_{0} t}\|\widetilde{\theta}(0)\| \\
& +k_{0} e^{-\lambda_{0} t}\left[N \lambda_{\max }\right] s_{\max , i}(t) \frac{e^{\lambda_{0} t}-1}{\lambda_{0}} \\
\leq & \bar{\kappa}\|\widetilde{\theta}(0)\|+\frac{k_{0}}{\lambda_{0}} \beta_{s}(t) .
\end{aligned}
$$

Considering the following equation consequently:

$$
\begin{aligned}
\int_{0}^{t} e^{\bar{a}_{\theta}(t-\tau)} L^{-1} S d \tau= & \int_{0}^{T_{1}} e^{\bar{a}_{\theta}(t-\tau)} L^{-1} S d \tau \\
& +\int_{T_{1}}^{t} e^{\bar{a}_{\theta}(t-\tau)} L^{-1} S d \tau
\end{aligned}
$$

and we will get

$$
\begin{aligned}
\|\tilde{\theta}(t)\| \leq & \bar{\kappa}\|\tilde{\theta}(0)\|+\int_{0}^{T_{1}} e^{\bar{a}_{\theta}(t-\tau)}\left\|L^{-1} S\right\| d \tau \\
& +\int_{T_{1}}^{t} e^{\bar{a}_{\theta}(t-\tau)}\left\|L^{-1} S\right\| d \tau \\
\leq & \bar{\kappa}\|\tilde{\theta}(0)\|+\frac{k_{0}}{\lambda_{0}} e^{-\lambda_{0} t}\left(e^{\lambda_{0} T_{1}}-1\right) \beta_{s}\left(T_{1}\right) \\
& +\frac{k_{0}}{\lambda_{0}} e^{-\lambda_{0} t}\left(e^{\lambda_{0} t}-e^{\lambda_{0} T_{1}}\right) \beta_{s T_{1}} \\
\leq & \bar{\kappa}\|\tilde{\theta}(0)\|+\frac{k_{0}}{\lambda_{0}}\left(\beta_{s}\left(T_{1}\right)+\beta_{s T_{1}}\right) .
\end{aligned}
$$

This completes the proof, which indicates that the tracking error of the total system will converge to the neighborhood of the origin; that is to say, the pitch angle of each AUV can eventually reach the desired value, and simultaneously the each AUV will move at the common desired depth synchronously.

\section{Coordinated Depth Control Design by Using Adaptative Sliding Mode with Model Uncertainties}

In practical applications for AUVs, the parameters of the model always have uncertainties, which can affect the performance of the closed-loop system seriously. It is necessary to design control strategy to take into account these uncertainties which is the main contribution of this section.

Consider the control law described by (37) in the previous section; if the control gains $c, N$ and the model parameters a are not known, consider the following adaptive slide control law:

$\delta_{e}$

$$
\begin{aligned}
& =\widehat{c}\left[-T S-K \operatorname{sgn}(S)-\widehat{\mathbf{a} Y}-\widehat{N} \operatorname{sgn}(S)+\ddot{\theta}_{d}-\Lambda L q\right] \\
& =\widehat{c} X,
\end{aligned}
$$

where $X=\left[-T S-K \operatorname{sgn}(S)-\widehat{\mathbf{a} Y}-\widehat{N} \operatorname{sgn}(S)+\ddot{\theta}_{d}-\Lambda L q\right]$ and

$$
\begin{aligned}
& \dot{\widehat{\mathrm{a}}}_{j i}=k_{1 i} S^{T} L^{-1} y_{j} \\
& \dot{\hat{c}}_{i}=-k_{2 i} S^{T} L^{-1} X \\
& \dot{\hat{n}}_{i}=k_{3 i} S^{T} L^{-1} \operatorname{sgn}(S) \text {, }
\end{aligned}
$$

where $i=0,1, \cdots, n, j=1,2, \cdots, 5, K_{1}=$ $\operatorname{diag}\left(k_{10}, k_{11}, \cdots, k_{1 n}\right), K_{2}=\operatorname{diag}\left(k_{20}, k_{21}, \cdots, k_{2 n}\right)$, and $K_{3}=\operatorname{diag}\left(k_{30}, k_{31}, \cdots, k_{3 n}\right)$ are design parameters and their diagonal elements are all positive.

Theorem 5. Consider the system composed by a fleet of $n$ AUVs of the form (8), guided by the slide mode control laws (64) with the sliding mode surface defined by (33) and adaptation 
law described by (65). Then, the control system proposed solves the coordinated depth problem; that is, all the AUVs will move at the same common desired depth when this referred depth information can be available to at least one AUV and the graph induced by the AUVs has a directed spanning tree.

Proof. Consider the following Lyapunov candidate function:

$$
\begin{aligned}
V= & \frac{1}{2} S^{T} L^{-1} S+\sum_{j=1}^{5} \sum_{i=0}^{n} \frac{1}{2 k_{1 i}} \widetilde{\mathrm{a}}_{j i}^{2}+\sum_{i=0}^{n} \frac{1}{2 k_{2 i} c_{i}} \widetilde{c}_{i}^{2} \\
& +\sum_{i=0}^{n} \frac{1}{2 k_{3 i}} \widetilde{n}_{i}^{2},
\end{aligned}
$$

where $\widetilde{\mathrm{a}}_{j i}=\widehat{\mathrm{a}}_{j i}-\mathrm{a}_{j i}, \widetilde{c}_{i}=\widehat{c}_{i}-c_{i}, \widetilde{n}_{i}=\widehat{n}_{i}-n_{i}$ are estimation errors of $\mathrm{a}_{j i}, c_{i}$, and $n_{i}$. The derivative of (66) can be derived as

$$
\begin{aligned}
\dot{V}= & S^{T} L^{-1} \dot{S}+\sum_{j=1}^{5} \sum_{i=0}^{n} \frac{1}{k_{1 i}} \widetilde{\mathrm{a}}_{j i} \dot{\widehat{\mathrm{a}}}_{j i}+\sum_{i=0}^{n} \frac{1}{k_{2 i} c_{i}} \widetilde{c}_{i} \dot{\vec{c}}_{i} \\
& +\sum_{i=0}^{n} \frac{1}{k_{3 i}} \widetilde{n}_{i} \dot{\hat{\vec{n}}}_{i} .
\end{aligned}
$$

Substituting (34) and adaptation law (65) into (67), we will get

$$
\begin{aligned}
\dot{V}= & S^{T} L^{-1}\left[\mathbf{a Y}+b \widehat{c} X+n_{\theta}-\ddot{\theta}_{d}+\Lambda L q\right] \\
& +\sum_{j=1}^{5} \sum_{i=0}^{n} \widetilde{\mathrm{a}}_{j i} S^{T} L^{-1} y_{j}-\sum_{i=0}^{n} \frac{\widetilde{b}_{i}}{b_{i}} S^{T} L^{-1} X \\
& +\sum_{i=0}^{n} \widetilde{n}_{i} S^{T} L^{-1} \operatorname{sgn}(S)
\end{aligned}
$$

Consequently substituting control law (64) into (68), we can have

$$
\begin{aligned}
\dot{V} & =S^{T} L^{-1}[\mathbf{a Y}-T S-K \operatorname{sgn}(S)-\widehat{\mathbf{a} Y}-\widehat{N} \operatorname{sgn}(S) \\
& \left.+\ddot{\theta}_{d}-\Lambda L q+n_{\theta}-\ddot{\theta}_{d}+\Lambda L q\right]+\sum_{j=1}^{5} \sum_{i=0}^{n} \widetilde{\mathrm{a}}_{j i} S^{T} L^{-1} y_{j} \\
& +\sum_{i=0}^{n} \widetilde{n}_{i} S^{T} L^{-1} \operatorname{sgn}(S) .
\end{aligned}
$$

Simply manipulations will yield

$$
\begin{aligned}
\dot{V}= & S^{T} L^{-1}\left[-T S-K \operatorname{sgn}(S)-\widehat{N} \operatorname{sgn}(S)+n_{\theta}\right] \\
& +\sum_{i=0}^{n} \widetilde{n}_{i} S^{T} L^{-1} \operatorname{sgn}(S) .
\end{aligned}
$$

According to Assumption 2, we can get

$$
\dot{V} \leq-S^{T} L^{-1} T S-S^{T} L^{-1} K \operatorname{sgn}(S),
$$

and we can find $\dot{V}<0$ for all $s_{i} \neq 0$. According to Lemma 4 consequently, we can know that there is a time constant $T_{2}$ which satisfies

$$
\|\widetilde{\theta}(t)\| \leq \bar{\kappa}\|\widetilde{\theta}(0)\|+\frac{k_{0}}{\lambda_{0}}\left(\beta_{s}\left(T_{2}\right)+\beta_{s T_{2}}\right),
$$

and this completes the proof, so the system state would converge to the sliding mode surface $S(t)=0$ in finite time, all errors converge to zero, and the closed-loop system is stable. Then the pitch angle of each AUV can eventually reach the desired value; meanwhile, all the AUVs will move at the same desired depth synchronously.

\section{Simulation Results}

In this section, we present simulation examples to validate the theoretical results proposed in this work. We consider a group of AUVs modeled by (8) with hydrodynamic parameters shown in Table 1.

The control law designed did not consider the input saturation, while the actual AUV always has a rudder angle limit and in this paper we assumed that $\delta_{e i}<15^{\circ}$. Choose the adaptive sliding mode controller control gains: $\lambda_{z i}=0.05$, $\lambda_{i}=1, k_{i}=10$, and $t_{i}=0.05$, the initial depth of the three AUVs was 10 meters, 12 meters, and 8 meters, respectively, and the common desired depth is set to 20 meters. The simulation results are shown from Figures 4-9.

From Figure 4 we can see that all AUVs will move at the same common desired depth; specifically, the AUVs move to the desired depth more quickly under the control of adaptive sliding mode strategy. In the meantime, we can also find that the pitch angles of all AUVs can reach synchronization from Figure 5; moreover, when the AUVs move to the common desired depth, all the pitch angles tend to 0 degrees. Compared to the sliding mode strategy, the pitch angle of each AUV has a more quick convergent speed by using the adaptive sliding mode method. Figure 6 depicts the according rudder inputs for each AUV, and we can find that they also achieved synchronization behaviors. Figure 7 shows the evolving of the sliding mode surface of each controller; we can find that it will converge to zero in 12 and 6 seconds under the sliding mode and adaptive sliding mode, respectively, which shows the effectiveness of adaptive sliding mode strategy proposed specifically. Figures 8 and 9 show the coordinated tracking error of depth and pitch attitudes separately; they all tend to zero eventually.

In summary, due to the model parameter error, although the sliding mode control without adaptive mechanism is stable, there is always a steady-state error, and the adjustment time is also longer. With the introducing of adaptive parameter estimation algorithms, the system can estimate the linearization parameters online and converge to zero quickly. At the same time, it should be noted that the introducing of adaptive also makes the jitter frequency of the AUV rudder angle higher. This shows that the adaptive algorithm increases the system's overshoot while improving the steadystate accuracy performance, but because the rudder angle converges quickly, it will hardly affect the stability of the system. 
TABLE 1: The parameters of each AUV.

\begin{tabular}{|c|c|c|}
\hline Parameter & Definition & Value \\
\hline$m$ & mass & $1600 \mathrm{~kg}$ \\
\hline$\rho$ & density & $1019.2 \mathrm{~kg} / \mathrm{m}^{3}$ \\
\hline$T$ & thrust & $1078 N$ \\
\hline G & gravity & $15680 N$ \\
\hline$L_{a}$ & length & $7.738 m$ \\
\hline$S_{a}$ & maximum cross-sectional area & $0.224 m^{2}$ \\
\hline$J_{z}$ & moment of inertia around the $\mathrm{z}$-axis & $6771.8 \mathrm{~kg} \cdot \mathrm{m}^{2}$ \\
\hline$x_{c}$ & distance between center of mass and center of buoyancy & $0 m$ \\
\hline$y_{c}$ & distance of the center of mass move down & $-0.03 m$ \\
\hline$z_{c}$ & distance of the center of mass side shift & $0 m$ \\
\hline$K_{11}$ & vertical additional mass factor & 31.588 \\
\hline$K_{33}$ & lateral additional mass factor & 1554.672 \\
\hline$K_{35}$ & additional spacing factor & 276.113 \\
\hline$K_{55}$ & lateral additional moment of inertia factor & 6905.1545 \\
\hline$C_{y}^{\alpha}$ & positional derivative of lift factor versus angle of attack & 2.172 \\
\hline$C_{y}^{\delta_{e}}$ & positional derivative of lift factor versus horizontal rudder angle & 0.4994 \\
\hline$C_{y}^{\bar{\omega}_{z}}$ & positional derivative of lift factor versus angular velocity & 1.362 \\
\hline$m_{z}^{\alpha}$ & positional derivative of pitching moment factor versus angle of attack & 0.5892 \\
\hline$m_{\underline{z}}^{\tilde{\delta}_{e}}$ & positional derivative of pitching moment factor versus horizontal rudder angle & -0.7339 \\
\hline$m_{z}^{\bar{\omega}_{z}}$ & positional derivative of pitching moment factor versus angular velocity & -0.2659 \\
\hline
\end{tabular}

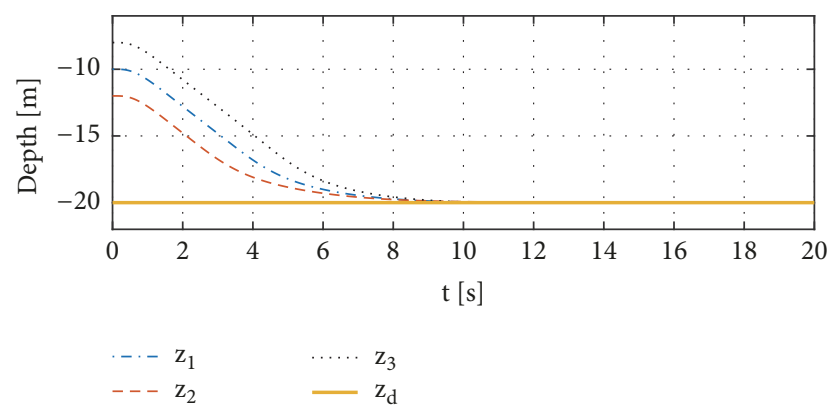

(a) Sliding mode control

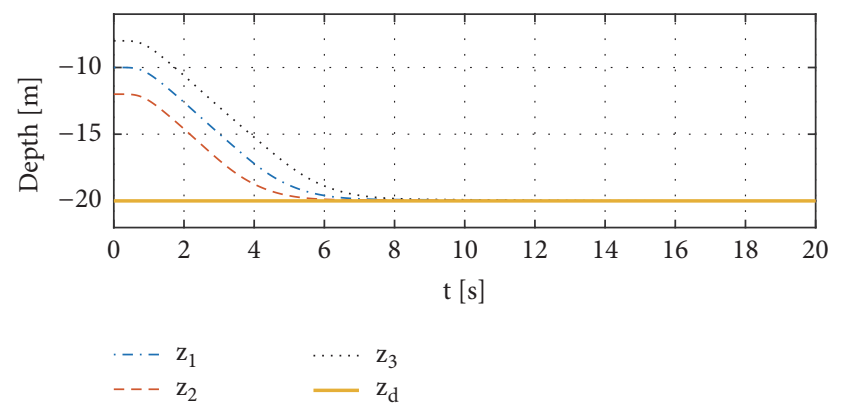

(b) Adaptive sliding mode control

FIGURE 4: The depth evolving of each AUV.

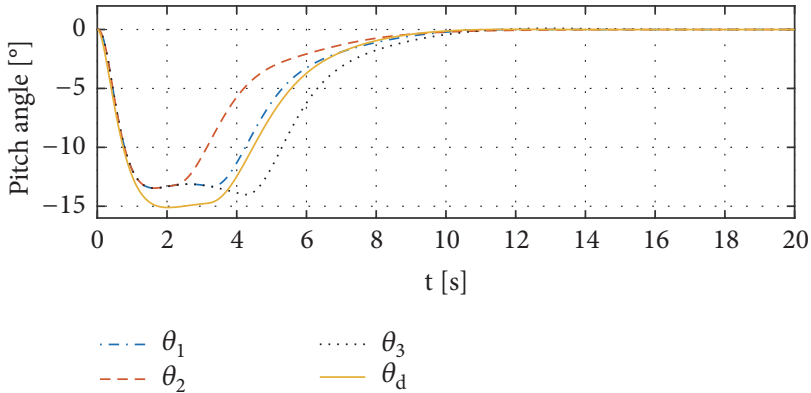

(a) Sliding mode control

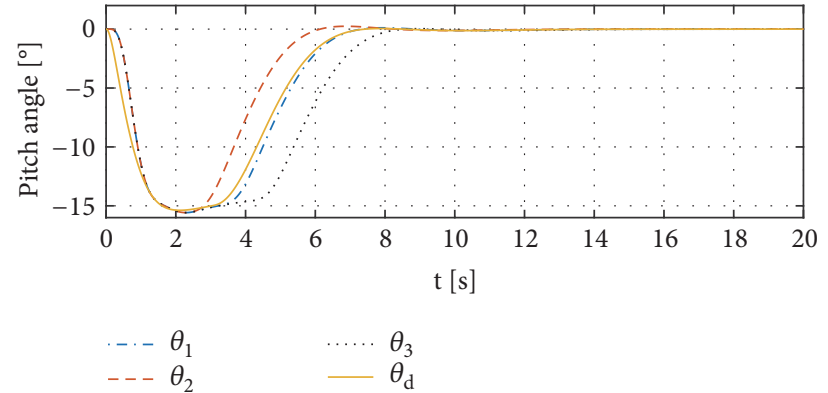

(b) Adaptive sliding mode control

FIGURE 5: Synchronized attitude of pitch angles of each AUV. 


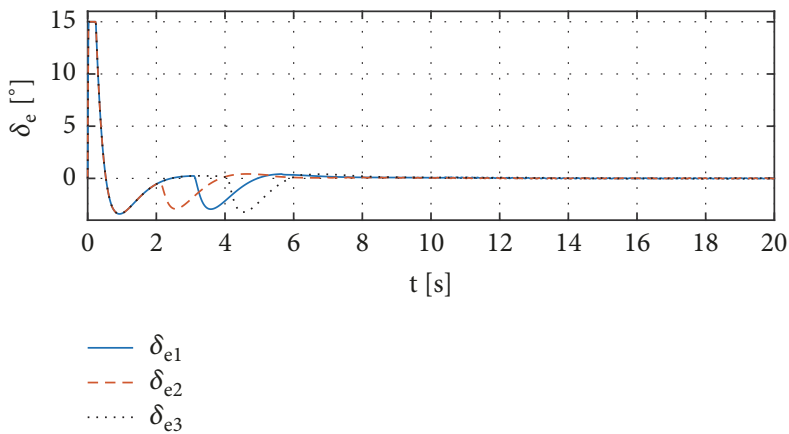

(a) Sliding mode control

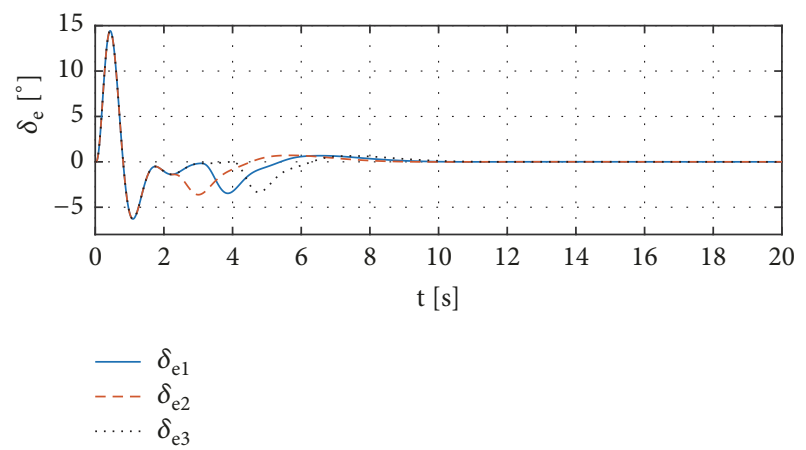

(b) Adaptive sliding mode control

FIgURE 6: The rudder input of each AUV.

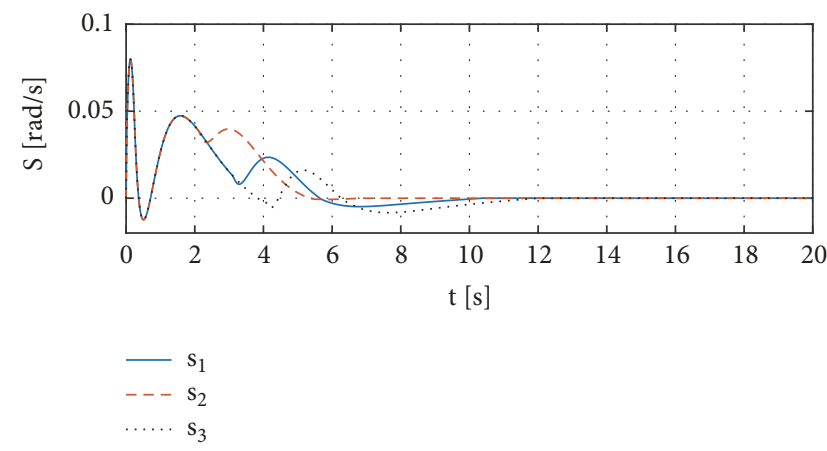

(a) Sliding mode control

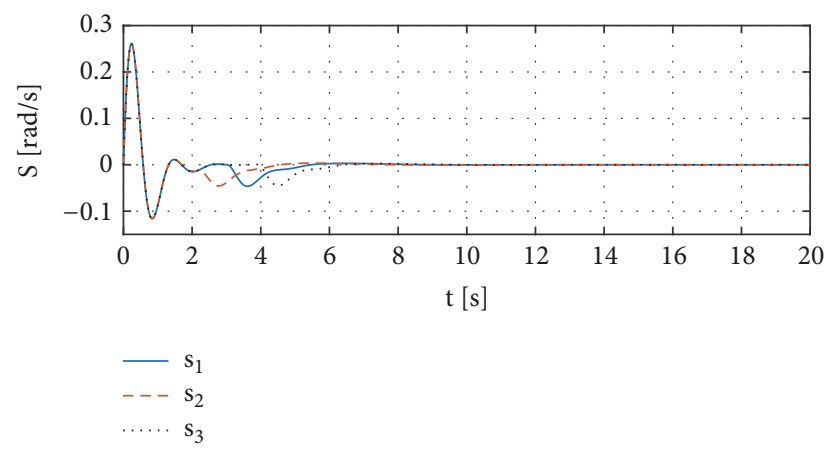

(b) Adaptive sliding mode control

FIGURE 7: The sliding mode surface of each controller.

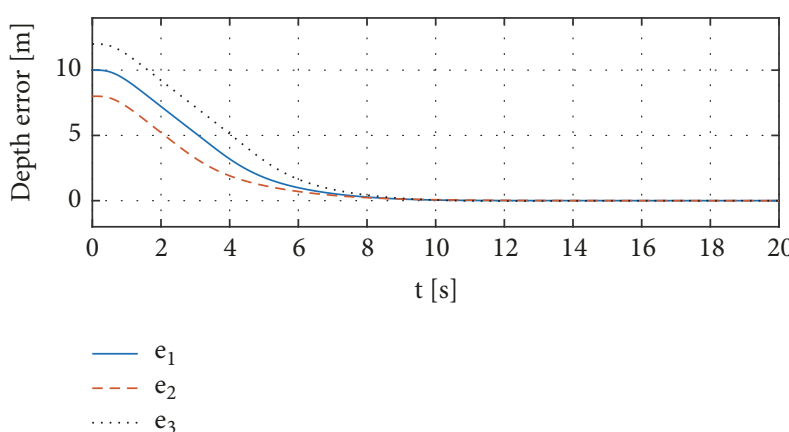

(a) Sliding mode control

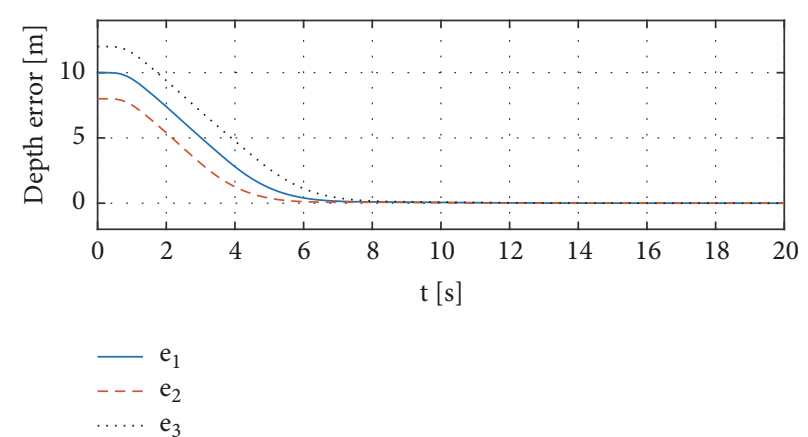

(b) Adaptive sliding mode control

FIGURE 8: The coordinated errors of depth tracking.

\section{Conclusion}

In this article, we discuss the coordinated depth control problem of multiple AUVs. We propose a distributed adaptive control law using the coordinated depth error based on sliding method; each AUV adopts deep-pitch double loop control. Our strategy is fully distributed because each controller only needs information of its neighbors and the desired common depth information can only be available to one subset of the AUVs. The uncertainty of the models is considered by adaptive laws. The stability of the control law proposed is analyzed and proved theoretically. The simulation results validate the effectiveness of algorithm proposed. We suppose that the topology between the AUVs is fixed and there are no communication delays. All the topics about multiple vehicles, such as dynamic changing topologies, time delays, and data dropouts, will warrant our future research.

\section{Data Availability}

The participant-level data of this work are the dynamic model parameters of AUV, which can be referred to Table 1 for details. 


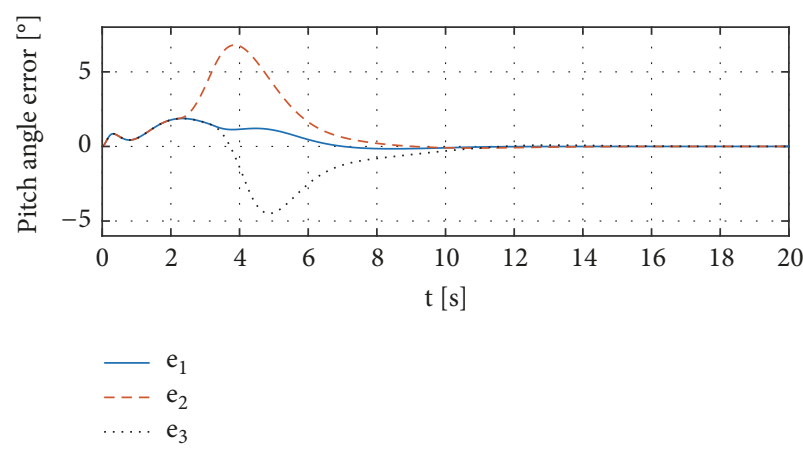

(a) Sliding mode control

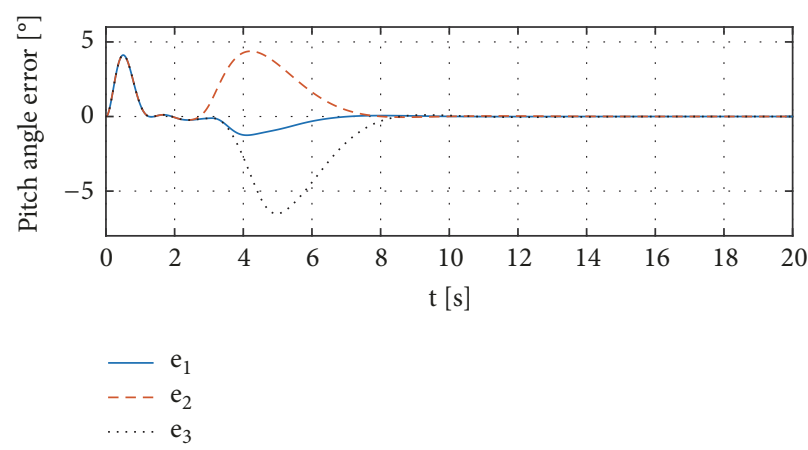

(b) Adaptive sliding mode control

FIGURE 9: The coordinated errors of pitch tracking.

\section{Disclosure}

Yintao Wang and Yani Zhang are the co-first authors.

\section{Conflicts of Interest}

The authors declare that there are no conflicts of interest regarding the publication of this paper.

\section{Acknowledgments}

This work was supported by the National Natural Science Foundation of China via Grants 51209175 and 61472326.

\section{References}

[1] C. Yang, X. Wang, L. Cheng, and H. Ma, "Neural-learningbased telerobot control with guaranteed performance," IEEE Transactions on Cybernetics, vol. 4, no. 10, pp. 2148-3159, 2017.

[2] C. Yang, K. Huang, H. Cheng, Y. Li, and C. Su, "Haptic identification by elm controlled uncertain manipulator," IEEE Transactions on Systems, Man, and Cybernetics: Systems, vol. 47, no. 8, pp. 2398-2409, 2017.

[3] C. Yang, X. Wang, Z. Li, Y. Li, and C. Su, "Teleoperation control based on combination of wave variable and neural networks," IEEE Transactions on Systems, Man, and Cybernetics: Systems, vol. 47, no. 8, pp. 2125-2136, 2017.

[4] T. Fossen, Guidance and control of ocean vehicles, JOHN WILEY, 1994.

[5] S. M. Zanoli and G. Conte, "Remotely operated vehicle depth control," Control Engineering Practice, vol. 11, no. 4, pp. 453-459, 2003.

[6] J.-H. Li and P.-M. Lee, "Design of an adaptive nonlinear controller for depth control of an autonomous underwater vehicle," Ocean Engineering, vol. 32, no. 17-18, pp. 2165-2181, 2005.

[7] W. M. Bessa, M. S. Dutra, and E. Kreuzer, "Depth control of remotely operated underwater vehicles using an adaptive fuzzy sliding mode controller," Robotics and Autonomous Systems, vol. 56, no. 8, pp. 670-677, 2008.
[8] J. Yang and S. Tian, "Research on depth control algorithm of underwater vehicle based on theory of sliding mode," Computer Measurement Control, 2017.

[9] W. Ren and R. W. Beard, "Consensus seeking in multiagent systems under dynamically changing interaction topologies," IEEE Transactions on Automatic Control, vol. 50, no. 5, pp. 655661, 2005.

[10] F. Xiao, L. Wang, and Y. Jia, "Fast information sharing in networks of autonomous agents," in Proceedings of the American Control Conference (ACC '08), pp. 4388-4393, 2008.

[11] M. Porfiri and D. J. Stilwell, "Consensus seeking over random weighted directed graphs," IEEE Transactions on Automatic Control, vol. 52, no. 9, pp. 1767-1773, 2007.

[12] L. Fang and P. J. Antsaklis, "Asynchronous consensus protocols using nonlinear paracontractions theory," Automatic Control, IEEE Transactions on, vol. 53, no. 10, pp. 2351-2355, 2008.

[13] Y. Cao and W. Ren, "Multi-vehicle coordination for doubleintegrator dynamics under fixed undirected/directed interaction in a sampled-data setting," International Journal of Robust and Nonlinear Control, vol. 20, no. 9, pp. 987-1000, 2010.

[14] G. Xie and L. Wang, "Consensus control for a class of networks of dynamic agents," International Journal of Robust and Nonlinear Control, vol. 17, no. 10-11, pp. 941-959, 2007.

[15] I. F. Ihle, M. Arcak, and T. I. Fossen, "Passivity-based designs for synchronized path-following," Automatica, vol. 43, no. 9, pp. 1508-1518, 2007.

[16] J. Almeida, C. Silvestre, and A. Pascoal, "Cooperative control of multiple surface vessels in the presence of ocean currents and parametric model uncertainty," International Journal of Robust and Nonlinear Control, vol. 20, no. 14, pp. 1549-1565, 2010.

[17] Y. Zhang, Y. Wang, and Y. Yao, "Distributed adaptive sliding mode control for attitudes synchronization of multiple autonomous underwater vehicles," Advances in Mechanical Engineering, vol. 9, no. 11, 2017.

[18] L. Zuo, R. Cui, and W. Yan, "Terminal sliding mode-based cooperative tracking control for non-linear dynamic systems," Transactions of the Institute of Measurement and Control, vol. 39, no. 7, pp. 1081-1087, 2017.

[19] J. Zhang, Laplacian and signless Laplacian matrices of graphs [Ph.D. thesis], Shang Hai Jiaotong University, 2014.

[20] R. Cui, B. Ren, and S. S. Ge, "Synchronised tracking control of multi-agent system with high-order dynamics," IET Control Theory \& Applications, vol. 6, no. 5, pp. 603-614, 2012. 
[21] X. Zhang, Attitude synchronization and formation control of Multi-AUV System [Ph.D. thesis], School of Marine Science and Technology Northwestern Polytechnical University, 2017.

[22] Z.-J. Wang, W.-S. Yan, J. Gao, and Q.-N. Zhang, "Adaptive sliding-mode control of tri-speed torpedo," Acta Armamentarii, 2012. 


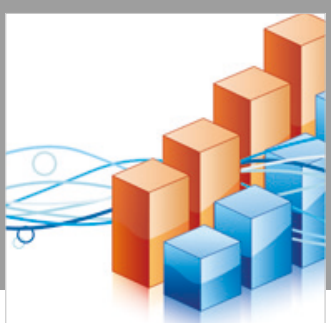

Advances in

Operations Research

\section{-n-m}
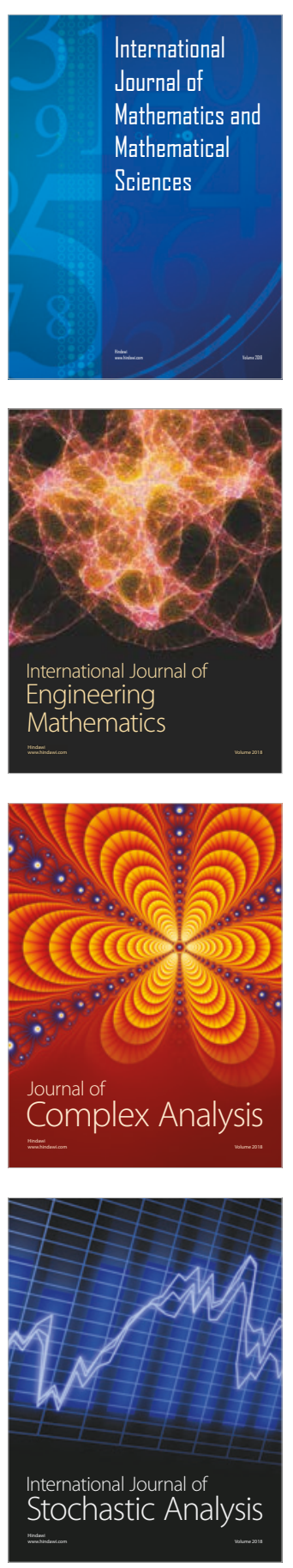
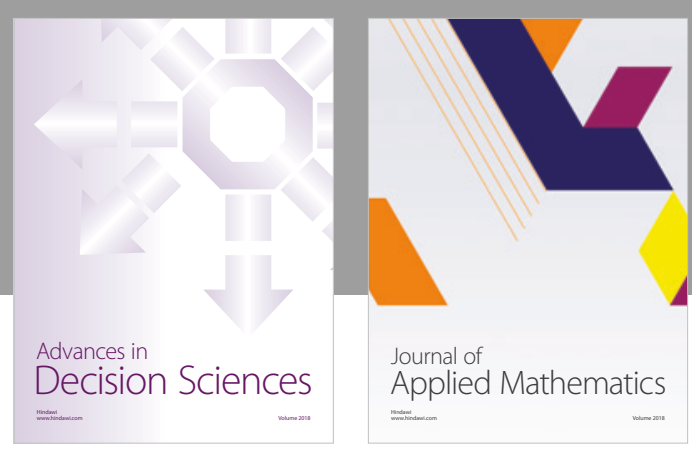

Journal of

Applied Mathematics
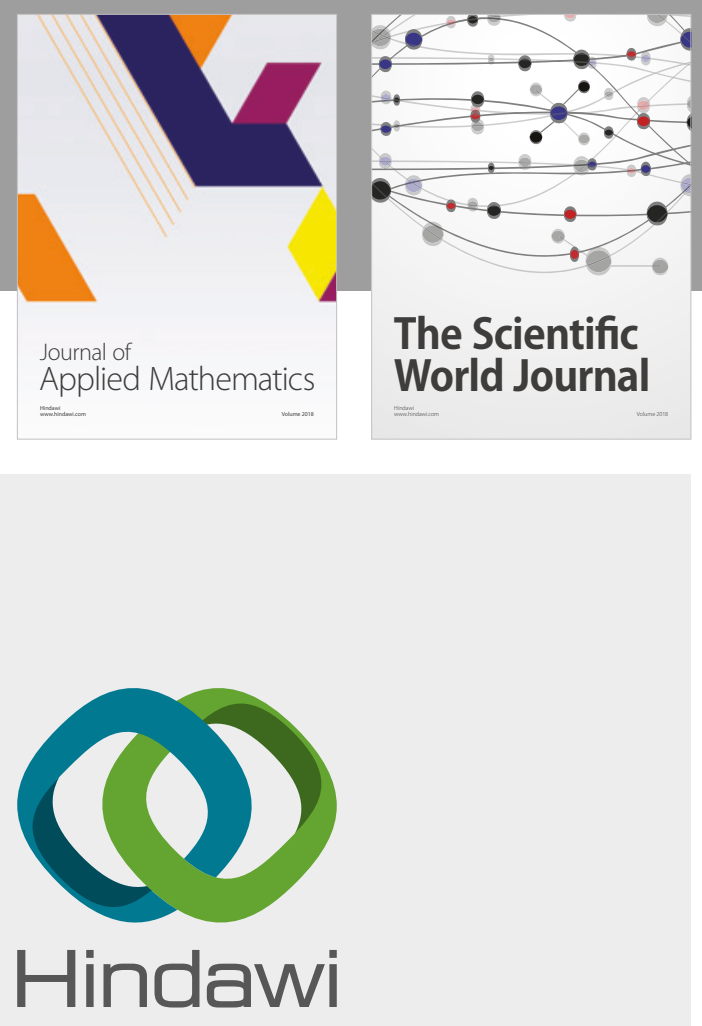

Submit your manuscripts at

www.hindawi.com

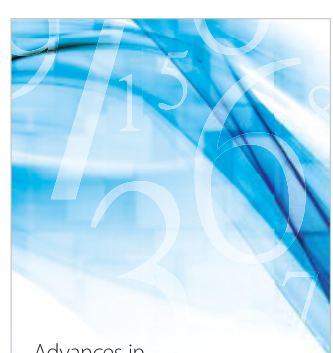

Advances in
Numerical Analysis
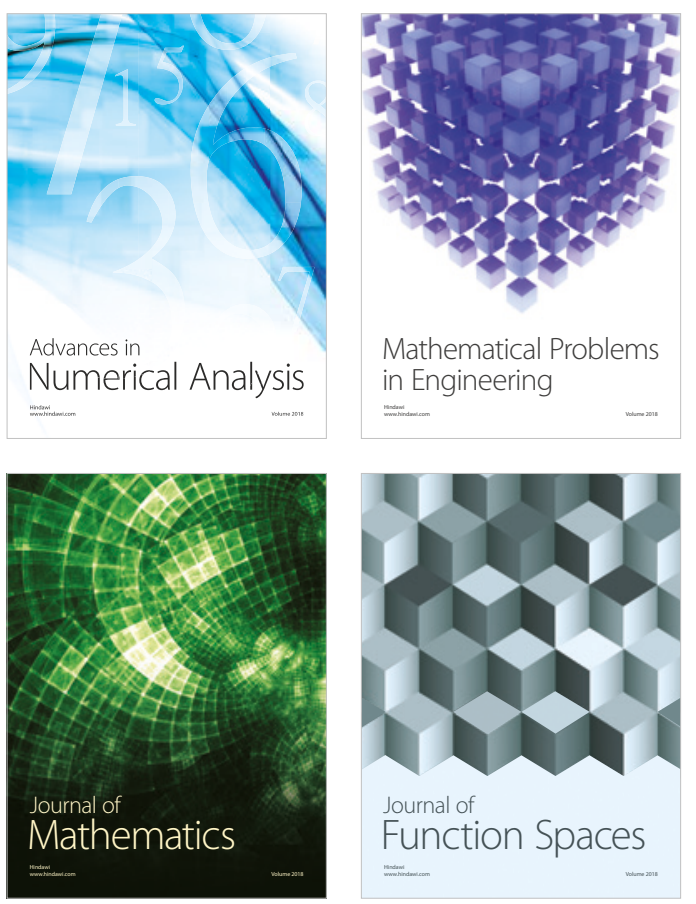

Mathematical Problems in Engineering

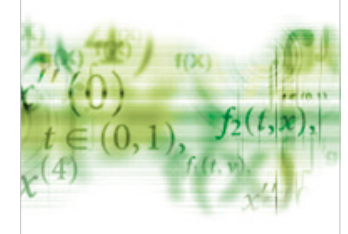

International Journal of

Differential Equations

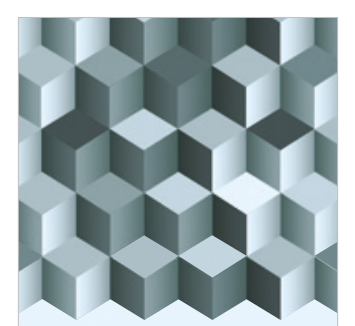

Journal of

Function Spaces
The Scientific

World Journal

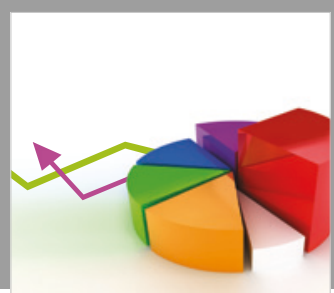

Journal of

Probability and Statistics
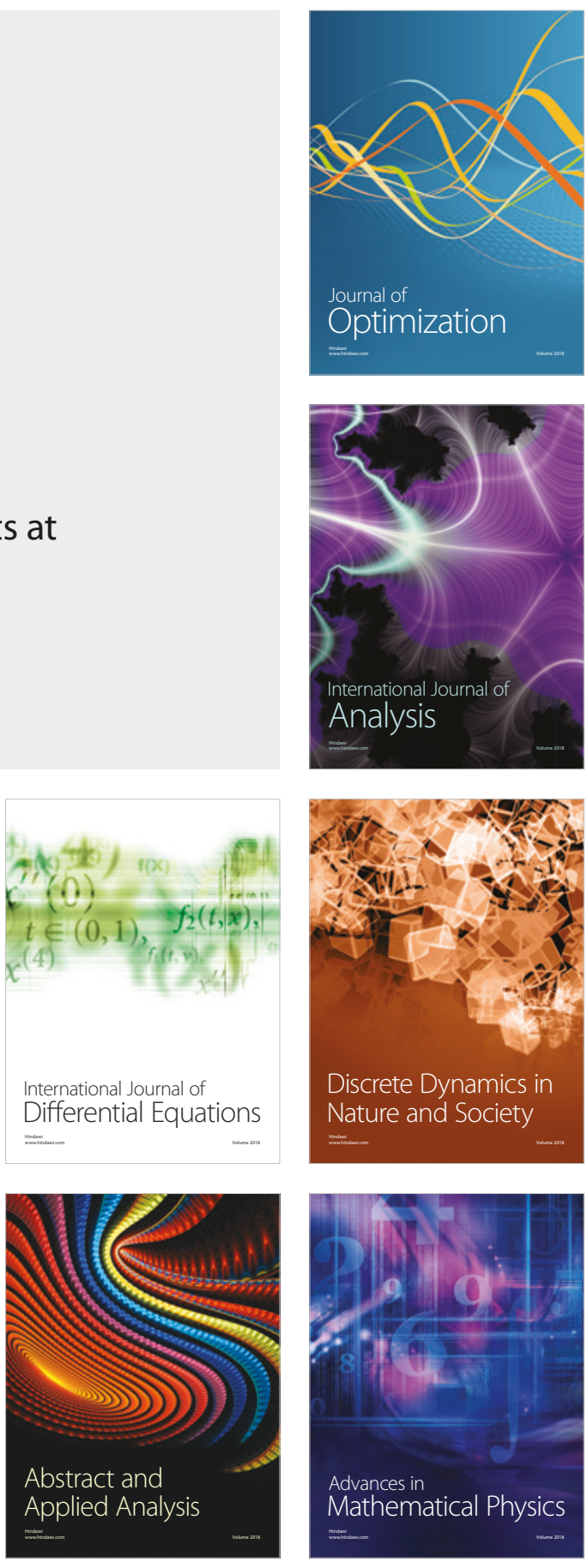